\title{
Drosophila PQBP1 Regulates Learning Acquisition at Projection Neurons in Aversive Olfactory Conditioning
}

\author{
Takuya Tamura, ${ }^{1}$ Daisuke Horiuchi, ${ }^{1}$ Yi-Chung Chen, ${ }^{2}$ Masaki Sone, ${ }^{1,3}$ Tomoyuki Miyashita,,${ }^{4}$ Minoru Saitoe, ${ }^{4}$ \\ Natsue Yoshimura, ${ }^{1}$ Ann-Shyn Chiang, ${ }^{2,5}$ and Hitoshi Okazawa ${ }^{1,6}$ \\ ${ }^{1}$ Department of Neuropathology, Medical Research Institute, Tokyo Medical and Dental University, Tokyo 113-8510, Japan, ${ }^{2}$ Institute of \\ Biotechnology, National Tsing Hua University, Hsinchu 30013, Taiwan, Republic of China, ${ }^{3}$ Department of Biomolecular Science, Faculty of \\ Science, Toho University, Chiba 274-8510, Japan, ${ }^{4}$ Tokyo Metropolitan Institute for Neuroscience, Tokyo 183-8526, Japan, ${ }^{5}$ Cold Spring Harbor \\ Laboratory, Cold Spring Harbor, New York 11724, and ${ }^{6}$ Core Research for Evolutional Science and Technology, Japan Science and Technology \\ Agency, Kawaguchi 332-0012, Japan
}

Polyglutamine tract-binding protein-1 ( $\mathrm{PQBP} 1)$ is involved in the transcription-splicing coupling, and its mutations cause a group of human mental retardation syndromes. We generated a fly model in which the Drosophila homolog of PQBP1 (dPQBP1) is repressed by insertion of piggyBac. In classical odor conditioning, learning acquisition was significantly impaired in homozygous piggyBac-inserted flies, whereas the following memory retention was completely normal. Mushroom bodies (MBs) and antennal lobes were morphologically normal in $\mathrm{dPQBP1-mutant}$ flies. Projection neurons (PNs) were not reduced in number and their fiber connections were not changed, whereas gene expressions including NMDA receptor subunit 1 (NR1) were decreased in PNs. Targeted double-stranded RNAmediated silencing of $A P Q B P 1$ in PNs, but not in MBs, similarly disrupted learning acquisition. NR1 overexpression in PNs rescued the learning disturbance of $\mathrm{APQBP} 1$ mutants. HDAC (histone deacetylase) inhibitors, SAHA (suberoylanilide hydroxamic acid) and PBA (phenylbutyrate), that upregulated NR1 partially rescued the learning disturbance. Collectively, these findings identify dPQBP1 as a novel gene regulating learning acquisition at PNs.

\section{Introduction}

Drosophila melanogaster is a powerful tool for analyzing mechanisms of learning and memory, and various methods have been developed to train a fly (Pitman et al., 2009). Aversive/appetitive olfactory conditionings and olfactory conditioning of probosis extension reflex are used for the analysis of olfactory memory. Courtship conditioning is based on experience-dependent suppression of male courtship behavior. Flight simulator induces visual or motor learning. Buridan's paradigm is for used spatial orientation memory. Especially, intensive studies by classical aversive olfactory conditioning led to classification of four memory components: short-term memory (STM), middle-term

Received March 15, 2010; revised Aug. 15, 2010; accepted Aug. 18, 2010.

This work was supported by Ministry of Education, Culture, Sports, Science and Technology Grant-in-Aid for Scientific Research on Priority Area 18023014, Japan Society for the Promotion of Science Grant-in-Aid for Scientific Research 18390254, and Grant-in-Aid for Scientific Research on Innovative Areas (Foundation of Synapse and Neurocircuit Pathology) and Core Research for Evolutional Science and Technology from Japan Science and Technology Agency (H.O.). We are grateful to Dr. Tina Rich (University of Glasgow, Glasgow, UK) for critical comments. We thank Tayoko Tajima for her excellent technical support in morphological analyses and Dr. E. Buchner (Theodor Boveri Institute für Biowissenshaften, Wurzburg, Germany) for anti-nc 82 antibody. Some of fly stocks were provided from National Institute for Genetics (Japan) or Bloomington Drosophila Stock Center (Indiana University, Bloomington, IN). GH146-LexA::GAD and LexAop-GFP fly stocks were kindly provided from Dr. Tzumin Lee (Janelia Farm Research Campus, Howard Hughes Medical Institute, Ashburn, VA) and Dr. Kei Ito (University of Tokyo, Tokyo, Japan).

Correspondence should be addressed to Hitoshi Okazawa, Department of Neuropathology, Medical Research Institute, Tokyo Medical and Dental University, 1-5-45, Yushima, Bunkyo-ku, Tokyo 113-8510, Japan. E-mail: okazawa-tky@umin.ac.jp.

DOI:10.1523/JNEUROSCI.1319-10.2010

Copyright $\odot 2010$ the authors $\quad$ 0270-6474/10/3014091-11\$15.00/0 memory (MTM), and long-term memory (LTM) including anesthesia-resistant memory (ARM) and long-lasting synthesisdependent long-term memory (LLTM). It is generally believed that mushroom bodies (MBs) store STM, MTM, and ARM. Hence, most studies have been focusing on the plasticity of MBs and the memory genes expressed in MBs (for review, see Keene and Waddell, 2007). It is generally believed that conditioned stimuli (CSs) and unconditioned stimuli (USs) are combined in third-order olfactory pathway neurons, Kenyon cells in MBs, and the plasticity of these cells critically regulates memory formation in classical olfactory conditioning (Heisenberg, 2003).

However, several recent reports have suggested other neurons might also regulate learning and memory. Wu et al. (2007) revealed a critical role of cholinergic neurons in ellipsoid body for consolidation of LTM. However, a live imaging study expressing $\mathrm{pH}$-sensitive vesicular fluorescent protein, synapto-pHluorin in projection neurons (PNs) suggested a plasticity of PNs (Yu et al., 2004) that might lead to memory formation. There is an increase in literature that suggests that PNs perform an important computation that transforms the representation in antennal lobes (ALs) to a sparser one in MBs (for review, see Masse et al., 2009). This transformation is thought to underlie sensory coding in MBs.

NMDA receptors (NMDARs) are related to memory formation by aversive olfactory learning (Lin, 2005; Xia et al., 2005). Drosophila NMDAR subunit 1 (dNR1) mutants showed impaired learning (Xia et al., 2005), whereas MBs-specific knockdown of dNR1 disrupted $3 \mathrm{~h}$ memory but not learning acquisition ( $\mathrm{Wu}$ et al., 2007). However, dNR1 and dNR2 are 
necessary for LTM consolidation at ellipsoid body (EB) (Wu et al., 2007). Furthermore, EB-specific overexpression of NMDAR subunit 2 (dNR2) enhanced $24 \mathrm{~h}$ memory (Wu et al., 2007). These results indicate that NMDARs play different roles in learning acquisition and memory formation at multiple brain regions. Thus, NMDARs could also be responsible for learning acquisition formed outside of MB.

Polyglutamine tract-binding protein-1 (PQBP1), a binding protein to polyglutamine (polyQ) tracts (Waragai et al., 1999; Busch et al., 2003), was identified recently as a causative gene for mental retardation (MR) (Kalscheuer et al., 2003). An increasing number of clinical reports have indicated that PQBP1-linked MR syndromes have a high frequency (Stevenson et al., 2005) with recent data from the European Consortium of X-Linked MR suggesting that it might be almost equivalent to Rett syndrome (Poirier et al., 2006). Most PQBP-1 mutations induce truncation of C-terminal domain (CTD) by frameshift (Kalscheuer et al., 2003; Kleefstra et al., 2004; Lenski et al., 2004) or reduction of PQBP1 mRNA by non-sense decay (Kalscheuer et al., 2003). Conversely, in our analyses of transgenic mice expressing truncated PQBP1 cDNA, we detected a faint band of PQBP1 by Western blot analysis, although almost no PQBP1 was evident in the brain (our unpublished observation). The phenotype in learning and memory in human patients has not been sufficiently analyzed.

In this study, we generated a mutant fly model of PQBP1linked MR in which a transposon repressed the Drosophila homolog of PQBP1 (dPQBP1) gene expression and investigated their learning and memory by using the strength of Drosophila models (for review, see Restifo, 2005). Unexpectedly, we found that PQBP1 regulates aversive olfactory learning at PNs. In the mutant flies, reduction of $\mathrm{dPQBP} 1$ impaired learning acquisition without affecting specific memory components significantly, and the $\mathrm{PN}$-specific silencing of dPQBP1 by RNA interference (RNAi) led to a similar type of learning impairment. The number of PNs as well as the morphology of axonal and dendritic projections from PNs remained normal, whereas the PNs showed reduced expression of NMDAR subunit NR1 in the mutant flies. Collectively, these results suggested that functional impairment of PNs by $\mathrm{dPQBP} 1$ reduction underlies the loss of learning acquisition.

\section{Materials and Methods}

Fly stocks and rearing conditions. All flies were raised on a cornmeal medium without propionic acid and were maintained at $25^{\circ} \mathrm{C}$ and $60 \%$ humidity under a $12 \mathrm{~h} \mathrm{light/dark}$ cycle. The w; P\{FRT(whs) $2 \mathrm{~A}$ $\mathrm{P}\{$ neoFRT $\}$ 22B PBac\{GAL4D,EYFP $\}$ CG11820PL00109 (FlyBase stock number 19390) line generated by the group (Horn et al., 2003) was obtained from the Bloomington Drosophila Stock Center. The line has a transposable element, piggyBac inserted on the third chromosome, and two additional P-elements. To delete the extra P-elements, the mutant was outcrossed based on the yellow fluorescent protein (YFP) marker more than six generations with w1118 strain, w(CS10) (Dura et al., 1993). The resultant fly was designated a dPQBP1 mutant (supplemental Fig. 1, available at www.jneurosci.org as supplemental material). Then Cantonized w1118 strain was used as the wild-type (WT) control in this study. The UAS-dPQBP1 transgenic fly, carrying a $0.7 \mathrm{~kb}$ full-length cDNA of CG11820 on the second chromosome was developed from w1118 parental strain. GH146-LexA::GAD (LG-GH146) and LexAopGFP fly stocks were previously described (Lai et al., 2008).

Northern blot analysis. A total of $15 \mu \mathrm{g}$ of total RNA from fly heads was loaded in each lane and electrophoresed in a 1.2\% agarose/formaldehyde gel (SeaKem GTG; Takara) in MOPS (4-morpholinepropanesulfonic acid) buffer. Electrophoresis was performed at $30 \mathrm{mV}, 1 \mathrm{~h}$, and after 90 $\mathrm{mV}, 3 \mathrm{~h}$. Then the RNA was transferred to a nylon filter (Hybond-N+;
GE Healthcare) in $10 \times$ SSC overnight. The RNA was fixed onto the filter with a UV cross-linker. A total of $250 \mathrm{ng}$ of dPQBP1 cDNA fragment $\left(\sim 0.7 \mathrm{kbp}\right.$, full-length ORF) was labeled with $\left[\alpha^{-}{ }^{32} \mathrm{P}\right] \mathrm{dCTP}$ (GE Healthcare Bioscience) using a DNA labeling kit (Takara) and hybridized, and then analyzed with a BAS2000 (Fuji Film) and sequentially exposed to Kodak x-ray films at $-80^{\circ} \mathrm{C}$.

Semiquantitative reverse transcription- $P C R$. An oligo-dT primer was used for the reverse transcription (RT) reaction with $1 \mu \mathrm{g}$ of total RNA from fly heads. The cDNA was then amplified by PCR with both $\mathrm{APQBP} 1$ primer set (forward primer, 5'-ATGAGCCTGCCAGCTGCA-3', and reverse primer, 5'-CTAGTTATCATTACGGCGAC- $3^{\prime}$ ) and control dGAPDH1 primer set (forward primer, 5' -TCGGAATCAACGGATTTGGC-3', and reverse primer, 5'-GCTCTGCATATACTTGATCA-3'). Various numbers of PCR cycle were performed $(22,25,26$, and 30$)$, and a linear response was obtained at 25 cycles for both primer sets with a 1/400 volume of the cDNA (data not shown). Amplified products were resolved by electrophoresis with $1 \%$ agarose gels (SeaKem GTG; Takara), and then stained with ethidium bromide. Gel data was recorded by photographing UV illuminated bands, and then scanning. Scanned bands were quantified by NIH Scion Image with a computer.

Behavior test. All memory-related tests were performed in a conditioned environmental room in which flies performed olfactory conditioning at $25^{\circ} \mathrm{C}$ and $60 \%$ relative humidity under far-red light $(650 \mathrm{~nm}$, not detected by flies). Two aversive odors [3-octanol (OCT) and 4-methylcyclohexanol $(\mathrm{MCH})$; Fluka] were paired with electrical shock pulses of $60 \mathrm{~V} \mathrm{DC}$ with teaching machines used for all behavioral analyses.

We used standard single-cycle training (Tully and Quinn, 1985) with minor modifications for early-phase memory. Approximately 100 flies were exposed sequentially to OCT or MCH for $60 \mathrm{~s}$ with $45 \mathrm{~s}$ rest intervals after each odor presentation. During exposure to the first conditioned stimulus with shocks CS+ odor (either OCT or MCH), flies also received the unconditioned stimulus (US), $1.5 \mathrm{~s}$ pulses of electrical shocks, every $3 \mathrm{~s}$ for $1 \mathrm{~min}$. For $0 \mathrm{~h}$ memory test, the trained flies were tapped into the choice point of a T-maze in which they were exposed simultaneously to the CS+ and CS - (conditioned stimulus without shocks). For 1 and $3 \mathrm{~h}$ memory test, they were kept in food vials until examination. As previously described (Tully et al., 1994), a performance index (PI) was calculated so that a 50:50 distribution (no memory) yields a PI of zero and a 0:100 distribution away from the CS + yields a PI of 100 .

For $24 \mathrm{~h}$ memory test, spaced training, which induces both long-lasting anesthesia-resistant memory and de novo protein synthesis-dependent longterm memory (LLTM), was used. As described previously (Yoshimura et al., 2006), flies received five training cycles with a $1 \mathrm{~h}$ rest interval between each. Then the flies were tested at $24 \mathrm{~h}$ after the last training, and the PI was calculated in the same fashion as single cycle training.

Olfactory acuity test has performed as a two choice test. The concentrations of odors used were pure or 10 and 100 times diluted with mineral oil. Flies were placed at the choice point of the T-maze and allowed to choose an odor and pure air for $1 \mathrm{~min}$. The avoidance index was calculated in the same fashion as PI using odor instead of CS + . Shock reactivity of flies was performed as described previously (Tully and Quinn, 1985).

Morphology and immunostaining. Whole-mount preparations were processed, and confocal imaging of fly brains was performed according to the previous method (Chiang et al., 2001) with minor modifications. In brief, dissected brains were fixed with $4 \%$ paraformaldehyde in PBS for $2 \mathrm{~h}$ and fixed again with $4 \%$ paraformaldehyde in PBS containing $0.25 \%$ Triton X-100 for another $2 \mathrm{~h}$. Fixed tissues were then cleared by direct incubation in a drop of FocusClear (Pacgen) and then mounted in the MountClear (Pacgen) or VectaShield Mounting Medium (Vector Laboratories). The frontal sections were obtained from the whole-mount brain in which anterior side of AL was placed upward. Whole-mount brains were imaged with a Zeiss LSM 510 confocal microscope (Carl Zeiss) using a $40 \times$ C-Apochromat water-immersion objective lens [numerical aperture (NA), 1.2]. The "votex" module in Amira 2.3 (TGS) was used for volume rendering and rotation of volume images.

For the whole-mount immunostaining, adult fly brains were fixed with $4 \%$ paraformaldehyde in PBS, washed with $0.2 \%$ Triton-X in PBS, blocked with $5 \%$ skim milk- $0.2 \%$ Triton-X in PBS, and incubated with anti-nc82 (a generous gift from Dr. E. Buchner, Theodor Boveri Institute 

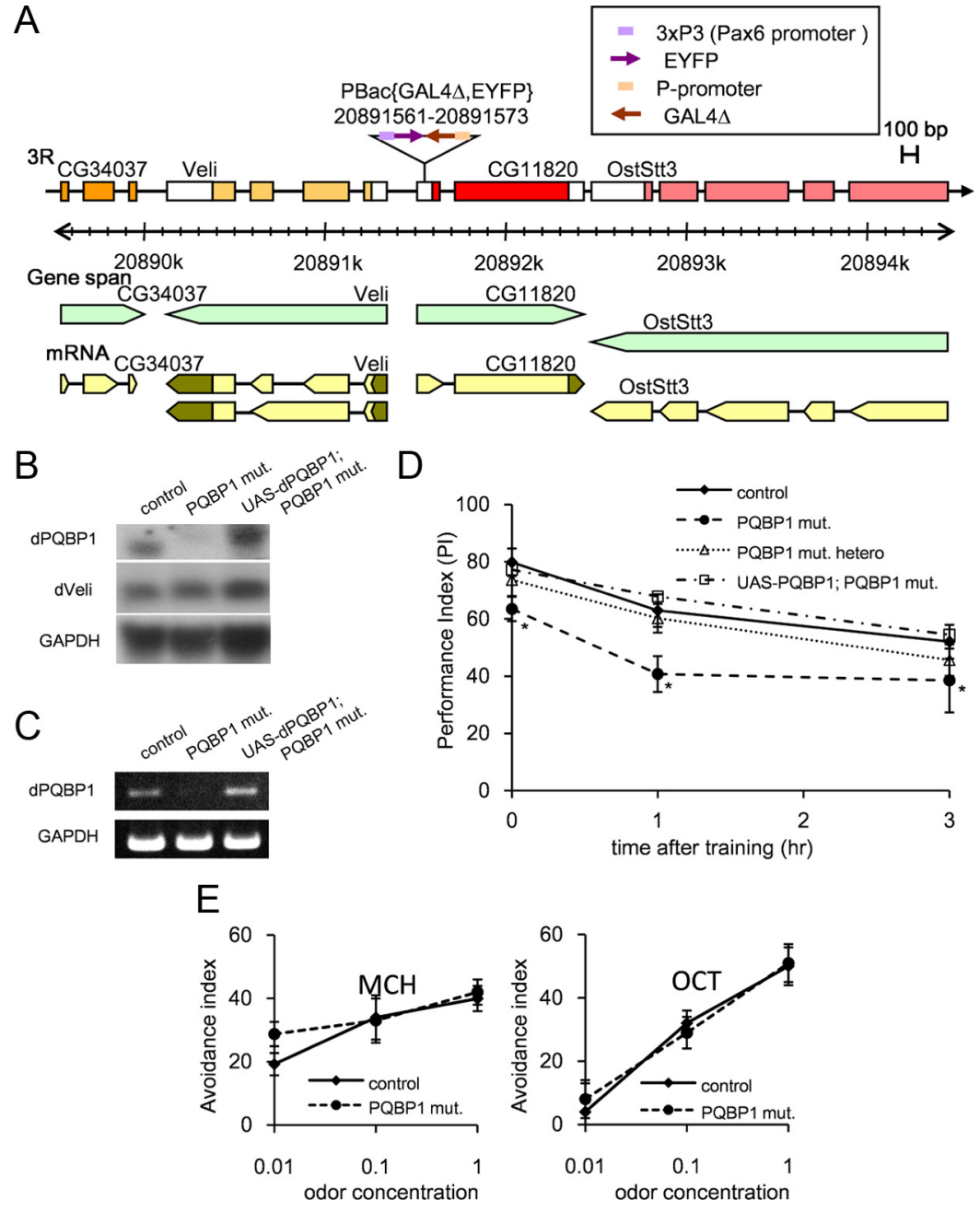

Figure 1. $\quad A P Q B P 1$ is a novel gene regulating learning acquisition. $A$, Schematic structure of $d P Q B P 1$ and surrounding genes. The $\mathrm{dPQBP} 1$-mutant genome contains a piggyBac transposon in the $5^{\prime}$-UTR of $\mathrm{APQBP} 1$. The piggyBac includes EYFP under control of pax6 promoter and GAL4 $\Delta$ under control of a p-promoter, as indicated. $\boldsymbol{B}$, Northern blot analysis of $\mathrm{dPQBP} 1$ (top) and a neighboring gene, Veli (middle). In the homozygous dPQBP1 mutant (PQBP1 mut), dPQBP1 mRNA was substantially reduced. The size of $\mathrm{APQBP} 1$ is larger because the transposon is positioned within an exon. In the rescue line (UAS-dPQBP1; PQBP1 mut), dPQBP1 mRNA was recovered, and the expression level of the transgene was higher than the endogenous level. No significant difference of Veli expression was observed among the genotypes. The band indicates Veli variant a, but we could not observe the b variant. $\boldsymbol{C}$, Semiquantitative RT-PCR supports the result of Northern blot. $\boldsymbol{D}$, The homozygous $\mathrm{APQBP1}$-mutant flies shows significantly lower PI than control (wild-type, w1118) flies. Their memory retention is normal since the curves decline in parallel. The curve of heterozygous flies is similar to controls. The PI and curve are completely recovered in the rescue flies. Mean $\pm \mathrm{SE}(n \geq 8)$ are shown at each time point and genotypes. The asterisks indicate significant differences $(p<0.01)$ by Welch's $t$ test between control and dPQBP1 mutant at each time point. $\boldsymbol{E}$, Olfactory acuity is not changed in the homozygous $\mathrm{APQBP} 1$ mutant. $\mathrm{MCH}$ and $0 \mathrm{CT}$ indicated in each graph are odors used for memory test. Avoidance index is plotted in a semilog graph. Mean $\pm \mathrm{SE}(n=6 \sim 8)$ are shown at each odor concentration in each genotype.

für Biowissenshaften, Wurzburg, Germany; diluted at 1:20) or anti- $\beta$ galactosidase (Promega; 1:200) for $36 \mathrm{~h}$, followed by Cy3- or Cy5conjugated secondary antibodies (Jackson ImmunoResearch; 1:50) and 4',6'-diamidino-2-phenylindole (DAPI) (Dojindo; 1:5000) for $12 \mathrm{~h}$.

Total number of PNs in GFP wild-type and GFP; dPQBP1-mutant flies were counted from images of serial horizontal sections at $2 \mu \mathrm{m}$ thickness, as described previously (Okada et al., 2009). The total GFP signal intensities of AL at the same $z$-axis level (the maximum diameter level) were quantified by LSM image browser (Carl Zeiss) in three flies of each genotype. The GFP signal of non-AL neuropil on the same section was subtracted as background.

For the paraffin section staining, proboscis were removed from dissected adult female fly heads and fixed in Carnoy's solution (ethanol/ chloroform/acetate, 6:3:1) for $1 \mathrm{~h}$ on ice, dehydrated in serial dilutions of ethanol, and embedded in paraffin (pathoprep546; melting point, $54 \sim 56^{\circ} \mathrm{C}$; Wako). The frontal sections $(6 \mu \mathrm{m})$ were deparaffinized and then stained with anti-NR1 (Xia et al., 2005) (diluted 1:100) Cy5-conjugated secondary antibodies (Jackson ImmunoResearch; 1:50) and DAPI (Dojindo; diluted 1:5000) for overnight. Confocal images were obtained with a Zeiss LSM 510 confocal microscope (Carl Zeiss) using a $20 \times$ C-Apochromat dry objective lens (NA, 0.8). For the frozen section staining, adult heads were fixed in $4 \%$ paraformaldehyde in PBS for $1.5 \mathrm{~h}$, incubated in 5 and $10 \%$ sucrose in PBS for 30 min, 15 and 20\% sucrose in PBS for $1 \mathrm{~h}$, and $30 \%$ sucrose in PBS overnight at $4^{\circ} \mathrm{C}$. After the heads were frozen in the OCT compound (Tissue-Tek), $10 \mu \mathrm{m}$ frontal sections were cut with a cryostat microtome. They were then stained with anti-ELAV (Developmental Studies Hybridoma Bank; diluted 1:50), anti-NR2 (Wu et al., 2007) (diluted 1:1000), Cy3-conjugated secondary antibodies (Jackson ImmunoResearch), and DAPI (Dojindo; diluted 1:5000).

Thermosensitive rescue with Gal8O ${ }^{\text {ts }}$. tubGal80 ${ }^{\text {ts }}$; $\mathrm{APQBP} 1^{\text {mut }}$ males were crossed with WT (for control), $d P Q B P 1^{\text {mut }}$ (for mutant), and UAS-dPQBP1; $A P Q B P 1^{\text {mut }}$ (for rescued) virgin females, respectively. Then mated flies were allowed for egg laying under permissive $\left(30^{\circ} \mathrm{C}\right)$ or restrictive $\left(25^{\circ} \mathrm{C}\right)$ temperatures. The $\mathrm{F}_{1}$ flies were collected within $2 \mathrm{~d}$ after eclosion. Then the flies were moved to the different temperature and kept for another $6 \mathrm{~d}$ and tested. If temperature shift is not necessary, flies were tested within $5 \mathrm{~d}$ after eclosion.

Generation of $d P Q B P 1-R N A i$ flies. The UASds-dPQBP1 fly (11820R-1) was obtained from National Institute for Genetics (National Institute for Genetics Stock Center, Mishima, Japan) and crossed with GH146-GAL4, NP225 or c747GAL4, OK107-Gal4 flies for PN-specific or MBspecific knockdown of $\mathrm{dPQBP} 1$, respectively. Genetic background of 11820R-1 fly was not corrected to that of WT; instead, we analyzed the flies under a heterozygous background but with the corresponding genetic background controls.

NR1 rescue experiment. UAS-dNR1 transgenic fly was or will be described in detail elsewhere (T. Miyashita and M. Saitoe, unpublished observations). Briefly, the fulllength dNR1 gene flanked to UAS promoter was integrated into the second chromosome. The fly was crossed with the APQBP1 mutant, and the genotype of the fly tested is $w$; $U A S-d N R 1 /+; P Q B P 1$ mutant.

Pharmacological rescue experiment. For the pharmacological rescue, drug solutions at 10 times higher concentration were mixed with medium at 1:9. The final concentration is described in Figure $8 C-F$. The eggs were laid on the drug-containing medium; they then were grown and kept in the bottle until they were tested.

Real-time PCR. Total RNA was prepared from 10 whole flies (3 d of age) with RNeasy mini kit (QIAGEN) and reverse transcribed with SuperScript VILO (Invitrogen). Quantitative PCR analyses were performed with the 7300 Real-Time PCR System (Applied Biosystems) using the SYBR Green for dPQBP1 (for the primer design, see supplemental Fig. 2, available at www.jneurosci.org as supplemental material) and TaqMan probe for actin5C. The sequences were as follows: forward primer, CCGAGCGCGGTTACTCTTT; reverse primer, CAACAT- 
AGCACAGCTTCTCCTTGAT; and TaqMan probe, CCGCTGAGCGTGAAATCGTCCGT.

Statistics. We used Welch's $t$ test for comparison of two groups that were expected to have normal distributions. For multiple comparison, we used ANOVA and post hoc Dunnett's test.

\section{Results \\ dPQBP1 reduction impairs \\ learning acquisition}

Drosophila melanogaster expresses a single homolog of mammalian PQBP1 (dPQBP1), encoded by the CG11820 gene on chromosome 3 (Yoshimura et al., 2006). dPQBP1 lacks CTD, although both the WW domain (WWD) and CTD are conserved in the other lower animals (Caenorhabditis elegans) and plants (Arabidopsis) (Yoshimura et al., 2006).

We used a mutant fly line that contains a mutator element of the piggyBac transposon (Horn et al., 2003) at the $5^{\prime}$ untranslated region ( $5^{\prime}$-UTR) region of the first exon of CG11820 (Fig. $1 A$; supplemental Fig. 1A, $B$, available at www. jneurosci.org as supplemental material) as well as two additional P-elements. To standardize the background and to delete the extra P-elements, the mutant was outcrossed (based on the YFP marker) for more than six generations with w1118 strain, w(CS10) (Dura et al., 1993). Northern blot analyses of the resultant flies showed that the expression level of dPQBP1 but not of a neighboring gene, dVeli, is substantially repressed in mutants (Fig. $1 B$ ). Semiquantitative PCR supported the observation of repressed dPQBP1 gene expression (Fig. $1 C$ ) allowing us to designate this mutant as a dPQBP1 hypomorph. To confirm that the phenotypes observed in the APQBP1 mutant were attributable to downregulated $\mathrm{dPQBP} 1$, we generated rescue flies by crossing the mutant flies with the dPQBP1 overexpression flies under the control of an endogenous enhancer/promoter (Fig. 1B, C, lane 3). The rescue flies expressed a higher amount of $\mathrm{dPQBP} 1$ than the wild-type control (Fig. 1C).

Because the PQBP1 gene is responsible for human MR (Kalscheuer et al., 2003; Kleefstra et al., 2004; Lenski et al., 2004), we tested learning and memory retention in the mutant flies. To evaluate their memory, we used olfactory conditioning (Tully and Quinn, 1985) and found that the homozygous dPQBP1 mutant showed a statistically significant decrease in PI within 3 min after single training (Fig. $1 D$, dashed line). The PI was 63.5 in the dPQBP1 mutant, whereas it was 79.8 in control (wild type, $w 1118$ ) (Fig. $1 D$, solid line). To analyze memory retention in the mutant, we tested the memory at 1 and $3 \mathrm{~h}$ after training. We found that defects in 1 and $3 \mathrm{~h}$ memory were similar to the defect at $0 \mathrm{~h}($ Fig. $1 \mathrm{D})$. These defects were completely recovered by expressing dPQBP1 under the control of GAL4 $\Delta$ included in piggyBac (Fig. $1 \mathrm{D}$, dash-dotted line). These results clearly demonstrated that dPQBP1 expression above a certain threshold level is essential for learning acquisition. In addition, we confirmed that sensory thresholds for olfactory (Fig. $1 E$ ) and electric stimuli (supplemental Table 1, available at www.jneurosci.org as supple- mental material) were not changed in the homozygous dPQBP1 mutant.

\section{Morphological analyses of PNs and MBs}

Because of the general consensus that MBs play the most important role in olfactory memory (for review, see Heisenberg, 2003; Margulies et al., 2005), we analyzed the morphology of MBs in dPQBP1 mutants. Disruption of neurotransmission by expressing shibire in c747-positive Kenyon cells in MBs almost completely blocks olfactory memory (Dubnau et al., 2001). This clearly indicates that c747-positive cells in MBs are essential for olfactory memory. Therefore, we used a c747-Gal4 driver to examine MB morphology. We also used mCD8-GFP to localize the fluorescent protein to cell membranes. Consistent with previous reports (Yang et al., 1995), Kenyon cells in $\alpha$, $\beta$, and $\gamma$ lobes were clearly observed in c747-Gal4/mCD8-GFP control flies (Fig. 2). In whole-mount preparation, c747-Gal4-induced mCD8-GFP expression indicated no significant morphological change in the MBs of homozygous and heterozygous dPQBP1 mutants. No significant difference was detected in the area of $\alpha$ and $\beta$ lobes between wild-type (2759-2915 $\mu \mathrm{m}^{2} /$ hemisphere) and dPQBP1mutant flies (2813-2879 $\mu \mathrm{m}^{2} /$ hemisphere). For the area measurement, both lobes were arranged on the same plane. In agreement with our previous research (Tamura et al., 2003), the volume of MBs increased from day 3 to day 20 in all genotypes tested (Fig. 2).

Since the structure of MBs was intact in the mutant, we next examined the morphology of PNs by using the GH146 gene promoter. It was reported that odor stimuli evoked a synaptic response at 3 min after olfactory conditioning but persisted for $<10$ 
A

Day 3 adults
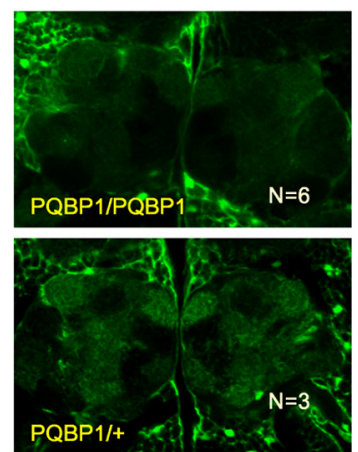

B
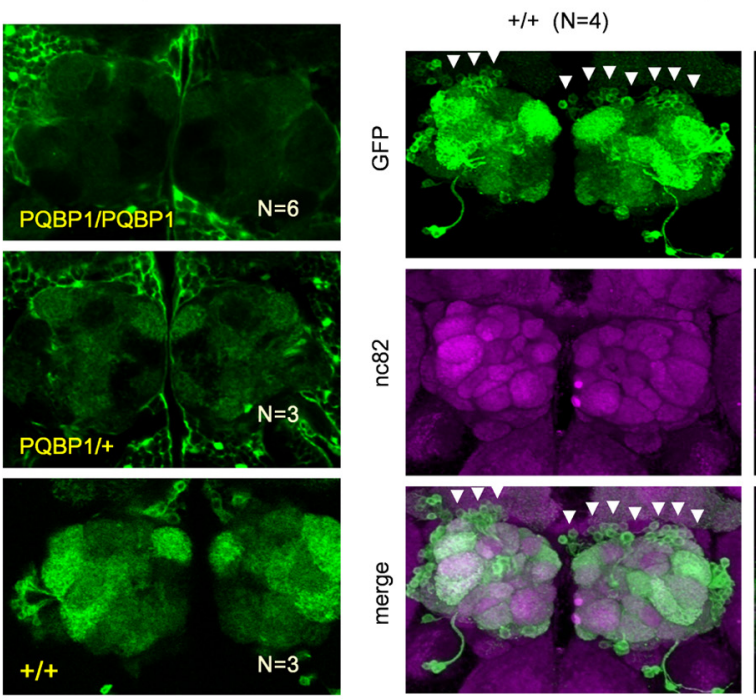

C

WT (NP225,GFP; +/+)

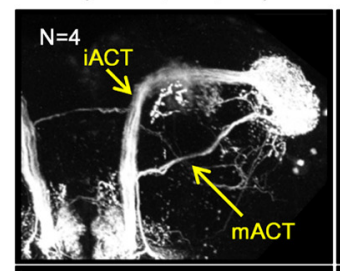

$\mathbf{N}=\mathbf{3}$

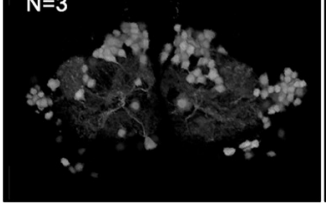
(NP225,GFP; PQBP1)

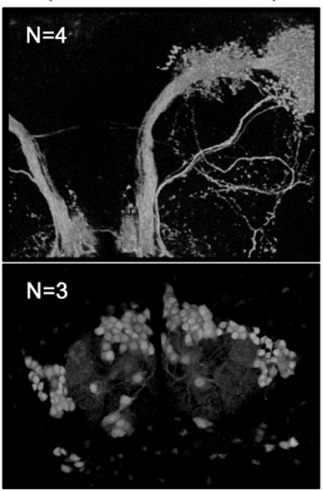

PQBP1 mut
Day 20 adults

PQBP1 mut $(\mathrm{N}=7)$
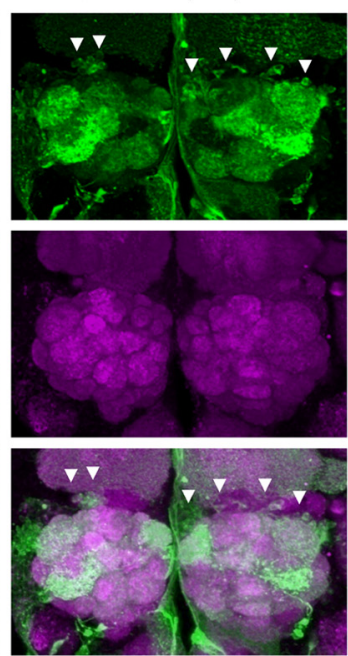

D
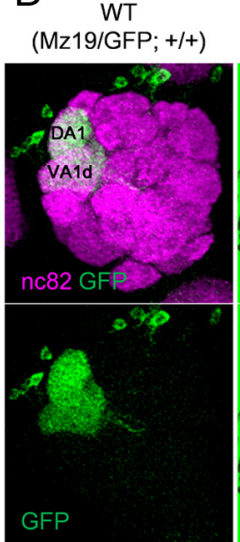

Figure 3. Effect of $d P Q B P 1$ reduction on PNs. mCD8-GFP was driven by projection neuron-specific drivers, GH146-Gal4 $(\boldsymbol{A}, \boldsymbol{B})$, and NP225 (C, D). A, At 3 d, GFP expression in the antennal glomeruli is reduced in homozygous (top) or heterozygous (middle) of $\mathrm{dPQBP1}$ mutant compared with wild-type flies carrying normal dPQBP1 (bottom). $\boldsymbol{B}$, Wild-type flies (left columns) and homozygous $\mathrm{dPQBP} 1$-mutant flies (right columns) at $20 \mathrm{~d}$. Expression of GFP is also significantly reduced in $\mathrm{PNs}$ (arrowheads) of $\mathrm{dPQBP1}$ mutants (top). Immunostains of nc 82 are not changed in $\mathrm{APQBP} 1$ mutants (middle), indicating that synapses of antennal glomeruli are normal in $\mathrm{APQBP} 1$ mutants. The bottom columns show merged images. The green and magenta signals indicate GFP and nc 82 , respectively. The arrowheads indicate "visible" cell bodies. C, Axonal projection patterns (top) and cell body patterns (bottom) are similar between genotypes. Both NP225 driver and mCD8-GFP are homozygous. mACT and iACT are indicated by arrows. $\boldsymbol{D}$, Confocal images of MZ19-Gal4 expression pattern show dendrite patterns (bottom) are similar between genotypes. MZ19-positive dendrites innervate DA1 and VA1d glomeruli in both WT and DPQBP1 mutant. The top panels show merged images of nc 82 (magenta) and GFP (green), and the bottom panels show GFP only images. The green signals in the background of mutant indicate YFP driven by pax6 promoter in the piggyBac transposon.

min in subpopulations of GH146-positive PNs (Yu et al., 2004). This result provided us a hypothesis that PNs might be relevant to learning acquisition in the aversive olfactory conditioning. PNs send dendrites to the AL (Heisenberg, 2003); thus, the GH146/ mCD8-GFP signal in the dendrites of PNs is found in $\mathrm{AL}$ (Berdnik et al., 2006). The mCD8-GFP signals in AL were significantly reduced in the homozygous $\mathrm{APQBP} 1$ mutant (Fig. $3 A, B$ ). The heterozygous $\mathrm{dPQBP} 1$ mutant showed an intermediate phenotype (Fig. 3A). These results suggested that certain functional or physical abnormalities of PNs occur in $\mathrm{DPQBP} 1$ mutants in a dose-dependent manner.

Olfactory information is relayed from olfactory receptor neurons (ORNs) to PNs that send dendrites to the glomeruli of $\mathrm{AL}$ (Jefferis et al., 2001). Correspondingly, elimination of ORNs but not of PNs is known to affect the size of the glomerulus (Berdnik

PQBP1 mut (Mz19/GFP; PQBP1)

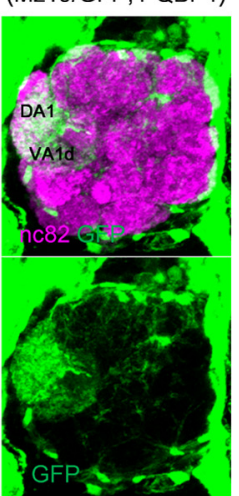

et al., 2006). Since immunohistochemistry with an antibody against a general presynaptic marker, nc82, showed that the size and signal intensity of glomeruli were similar in $\mathrm{dPQBP} 1$-mutant and control flies (Fig. 3B), ORNs were considered to be intact in the $\mathrm{APQBP} 1$-mutant flies.

\section{PNs are morphologically normal in dPQBP1 mutant}

Two hypotheses could explain the finding of diminished mCD8-GFP in PNs of dPQBP1-mutant flies. First, it may have been a physical abnormality for instance reduction of cell bodies and/or axonal/ dendritic projections of PNs. The second possibility was a functional abnormality of PNs reducing expression of $\mathrm{mCD} 8$ GFP. In the olfactory acuity test, $\mathrm{dPQBP} 1$ mutant showed completely normal thresholds of odor sensation (Fig. 1E).

To examine whether the number of PNs is changed, we analyzed PNs in GFP-labeled dPQBP1-mutant flies with another PNspecific driver, NP225 (Okada et al., 2009). We generated the flies carrying homozygous NP225 and UAS-mCD8-GFP in wild-type (NP225, UAS-mCD8-GFP; $+/+)$ or dPQBP1 homozygous (NP225, UAS-mCD8-GFP; PQBP1 mut) background (Fig. $3 C$ ). Although GFP signal was relatively weak in homozygous dPQBP1-mutant flies, no difference was found between wild-type and APQBP1mutant flies in the confocal microscopic analysis of inner antennocerebral tract (iACT) and medial antennocerebral tract (mACT) projection patterns (iACT and mACT) (Fig. 3C, top panels). Moreover, $\mathrm{PN}$ cell bodies were similar between the genotypes (Fig. 3C, bottom panels).

Quantitative analyses of the AL signal intensity and the number of NP225positive cell bodies reconfirmed our observation. The relative signal intensities on AL were significantly different $(p<$ $0.05, t$ test $)$ between wild-type $(1 \pm 0.12$; $n=3)$ and $\mathrm{dPQBP} 1$ mutant $(0.66 \pm 0.08$; $n=6)$ (mean $\pm \mathrm{SE}$ ). It is known that adult flies possess nearly 150 PNs and 100 local neurons (excitatory and inhibitory) around an AL (Stocker et al., 1997; Keene and Waddell, 2007), and approximately one-half of PNs (63-84/hemisphere) are NP225-positive in a wild-type fly (Okada et al., 2009). Our results showed that both the wild type and $\mathrm{PPQBP} 1$ mutant possessed similar numbers of NP225-positive PNs, 82.2 \pm 8.6 cells/hemisphere in wild type and $79.7 \pm 7.0$ cells/hemisphere in $\mathrm{dPQBP} 1$ mutant $(n=3$; mean $\pm \mathrm{SE}$ ). These results support the second possibility described above that PNs of $\mathrm{dPQBP} 1$ mutant possess a functional abnormality reducing gene expression, although their number was not decreased.

Next, we analyzed the dendritic innervations patterns of PNs in $\mathrm{APQBP} 1$ mutant. We used MZ19-Gal4, which drives expression in a small subset of PNs. The confocal analysis clearly 
Gal80ts/+; PQBP1 mut/+

Gal80/s/+; PQBP1 mut

Gal80!s/UAS-dPQBP1; PQBP1 mut
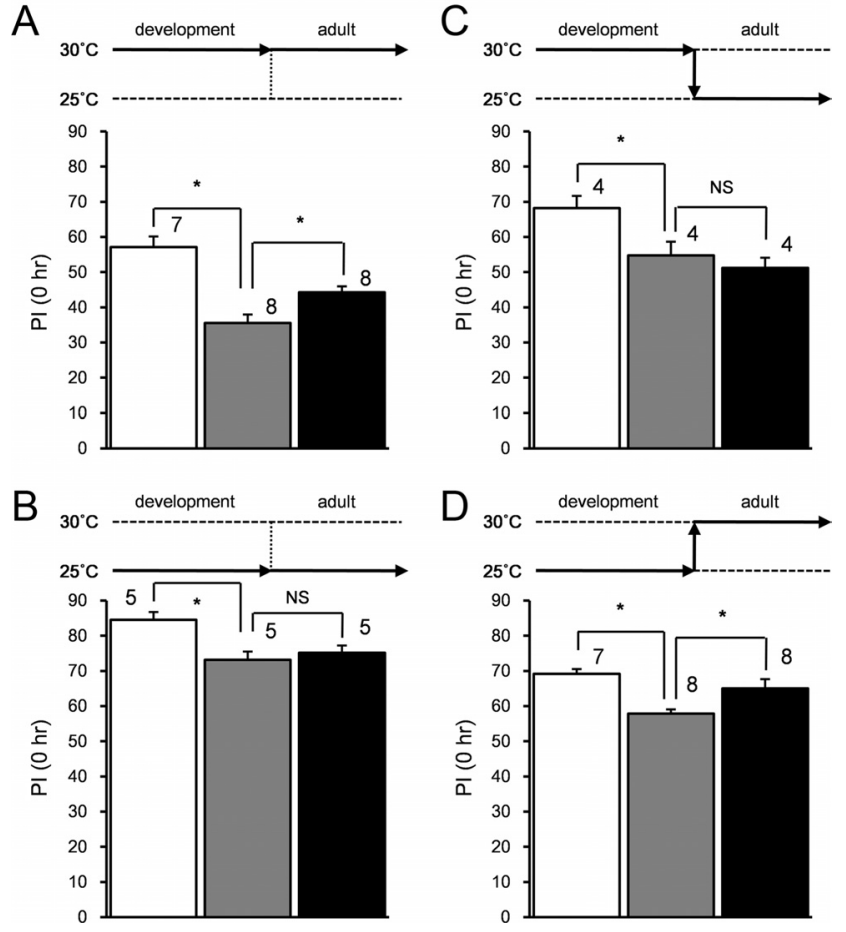

Figure 4. Expression of $\mathrm{APQBP} 1$ is necessary at adult fly. Genotypes are labeled at top of the panels: the open bar indicates heterozygous control, the gray bar indicates $\mathrm{APQBP} 1$ mutant, and the black bar indicates rescued flies. All genotypes carry heterozygous tublin-Gal $80^{\text {ts }}$ gene. Rearing conditions are shown as bold arrows above bar graphs. The $y$-axis of each graph is the performance index at $0 \mathrm{~h}$. $\boldsymbol{A}$, Flies were reared at $30^{\circ} \mathrm{C}$ throughout their life. $\boldsymbol{B}$, Flies were reared at $25^{\circ} \mathrm{C}$ throughout their life. $\mathrm{C}$, Flies were grown at $30^{\circ} \mathrm{C}$ during their development and adult flies were kept for $6 \mathrm{~d}$ at $25^{\circ} \mathrm{C}$ until the memory test. $\boldsymbol{D}$. Flies were grown at $25^{\circ} \mathrm{C}$ during their development and adult flies were kept for $6 \mathrm{~d}$ at $30^{\circ} \mathrm{C}$ until the memory test. Mean $\pm \mathrm{SE}$ are shown at each genotype. The asterisks between white and gray bars indicate significant difference from the controls ( $p<0.05$; we used Welch's $t$ test because the mutant should different from the heterozygous control at all temperature). The asterisks between gray and black bars indicate the significant rescue effect ( $p<0.05$; we used ANOVA and post hoc Dunnett's test to compare mutant with both WT and rescued flies). The sample sizes were shown above each bar.

showed that dendritic innervations patterns were not changed in dPQBP1 mutant. Dendrites of MZ19-positive PNs suitably innervate DA1 and VA1d glomeruli (Fig. $3 D$ ) similarly to a previous report (Lin et al., 2007).

During the experiment, we also noticed reduction of elav in homozygous dPQBP1 mutants around ALs (our unpublished observation) in addition to the reduction of GH146-driven mCD8-GFP. Elav has specific functions in neurons and the elav mutant is embryonic lethal (Soller and White, 2004); thereby, it is possible that the reduction of elav also leads to functional impairment of neurons.

\section{Expression of dPQBP1 is necessary at adulthood}

To elucidate whether the learning defect in $\mathrm{APQBP} 1$ mutant is caused by developmental defect or by neuronal dysfunction in adulthood, we used Gal80 ${ }^{\text {ts }}$ and performed a temporal rescue experiment by dPQBP1 similarly to a previous work (McGuire et al., 2003). When the flies were reared at $30^{\circ} \mathrm{C}$ (permissive temperature) from developmental stages to adulthood, learning defect in $\mathrm{dPQBP} 1$ mutant was significantly rescued (Fig. $4 \mathrm{~A}$, filled bar compared with gray bar). At $25^{\circ} \mathrm{C}$ (restrictive temperature),
Low mag
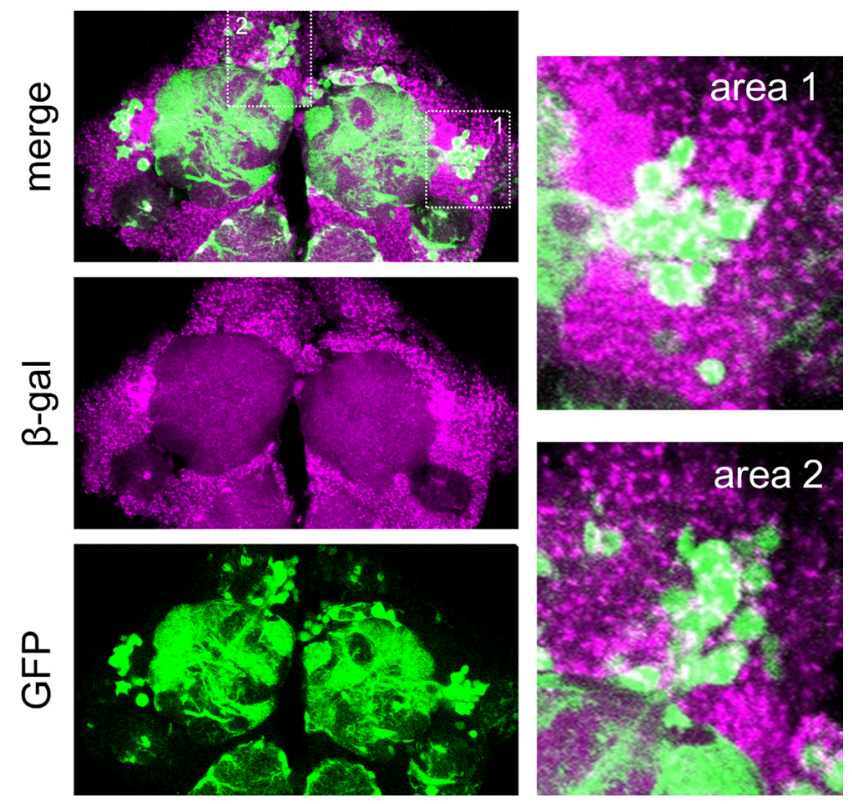

Figure 5. $\quad \mathrm{dPQBP1}$ gene expression detected by the endogenous promoter activity. Endogenous promoter activity of the $\triangle P Q B P 1$ gene was detected by LacZ in combination with LexA::GAD-GH146 induced GFP as a PN maker. Confocal microscopic images show that anti- $\beta$ Gal antibody (magenta, middle panel in Low mag) stains almost all the cell bodies surrounding antennal lobe (AL) and antennal nerve (AN). The GFP images (bottom panel in Low mag) are merged with $\beta$-Gal signals in the top panel. The right panels show enlarged views of PN cell bodies in the areas indicated by dotted line in the left top panel.

the learning defect was not rescued (Fig. 4B). For the temporal expression of dPQBP1 during development, flies were developed at $30^{\circ} \mathrm{C}$ and kept for $6 \mathrm{~d}$ at $25^{\circ} \mathrm{C}$. In this condition, the learning defect was not rescued, suggesting that loss of $\mathrm{dPQBP} 1$ during development did not affect the learning defect of adult flies (Fig. $4 C)$. However, when the mutant flies were developed at $25^{\circ} \mathrm{C}$ and kept for $6 \mathrm{~d}$ at $30^{\circ} \mathrm{C}$ for the temporal expression of dPQBP1 in adulthood, the learning defect was significantly rescued (Fig. $4 D)$. These results strongly suggested that the loss of dPQBP1 in adulthood is a critical factor for the defect of learning acquisition.

\section{dPQBP1 is expressed in PNs}

Next, we determined whether dPQBP1 is expressed in PNs. Although we generated more than eight polyclonal antibodies against synthetic peptides from various regions of $\mathrm{dPQBP} 1$, none of these was found to be useful for expression analysis (data not shown). Therefore, we made use of the finding that the mutator element of the dPQBP1 mutant contains GAL4 4 , a functional variant that is more stable than GAL4. GAL $4 \Delta$ is expressed under the control of endogenous enhancers in the vicinity of the transposon (i.e., enhancer trap system) (Horn et al., 2003). To determine the endogenous expression pattern of dPQBP1, we crossed dPQBP1 mutants with UAS-lacZ flies to observe LacZ expression driven by the dPQBP1 endogenous enhancer (Fig. 1A). Adult heads of the crossed $\mathrm{F}_{1}$ flies were stained with an anti $\beta$-Gal antibody. Our confocal images of whole brain showed that dPQBP1 was ubiquitously expressed in the adult CNS (our unpublished observation). To demonstrate the expression of dPQBP1 in PNs, we performed the $\beta$-Gal staining in combination with a LexA:GAD-GH146 line driving GFP as a marker of PNs. As the result, GFP-positive cell bodies were all $\beta$-Gal-positive (Fig. 5). The analysis sug- 


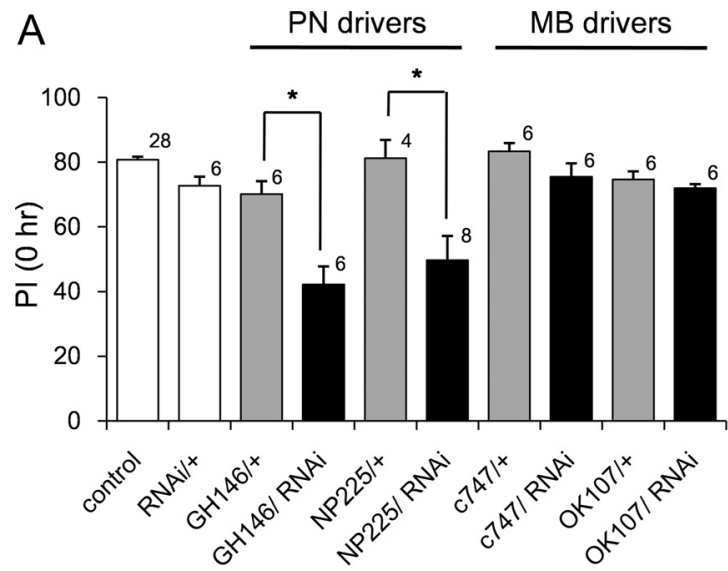

B
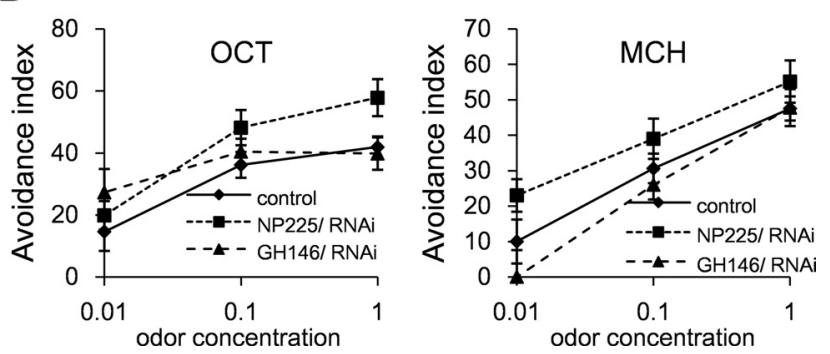

Figure 6. $\quad P N$-specific expression of $\triangle P Q B P 1-R N A i$ impairs learning similarly. $\boldsymbol{A}$, The graph shows $\mathrm{PI}$ at $0 \mathrm{~h}$ in various $\mathrm{DPQBP} 1$ knockdown flies. RNAi below the graph indicates a transgene of UAS-dPQBP1 RNAi (11820R-1). PN-specific knockdown of dPQBP1 (GH146/RNAi and NP225/ $\mathrm{RNAi}$ ) induced significant reduction of $\mathrm{PI}$ at $\mathrm{O} \mathrm{h}$ compared with driver alone flies (PN drivers). MB-specific silencing by C747-GAL4 and OK107 did not induce remarkable reduction of PI (MB drivers). Mean \pm SE are shown. The asterisk indicates significant difference between driver control and RNAi flies ( $p<0.01$; we used Welch's $t$ test for simple comparison of two groups). The number of experiments is shown above each bar. $\boldsymbol{B}$, Olfactory acuity is not changed in $\mathrm{dPQBP1}$ RNAi flies. MCH and OCT used in the memory test were examined. Avoidance indexes are plotted on a semilog graph. Mean $\pm \mathrm{SE}(n>4)$ are shown at each odor concentration and genotype.

gested that the cell bodies of the PNs around the ALs express dPQBP1.

\section{PN-specific suppression of dPQBP1 induces similar learning disturbance}

Our results mentioned above strongly suggested that $\mathrm{APQBP} 1$ is a gene involved in learning acquisition at the level of PNs. Further to test this idea, we next used an RNAi transgenic fly (UAS-dsdPQBP1) since we could not exclude the possibility that expression of $\mathrm{dPQBP} 1$ outside of PNs might also affect the learning acquisition. GH146 is expressed not only in PNs but also in MB anterior paired lateral (MB-APL) GABAergic neurons innervating MB lobes (Liu et al., 2009). Therefore, we also used another PN-specific driver, NP225 (Okada et al., 2009), to exclude possible involvement of MB-APL neurons. We crossed UAS-dsdPQBP1 flies with GH146-GAL4 or NP225 flies and examined its memory immediately after single training. The PI was significantly decreased in these PN-specific dPQBP1-RNAi flies when compared with the corresponding heterozygous driver flies (Fig. $6 A, \mathrm{GH} 146 /+, \mathrm{NP} 225 /+)$. The PI was similar among different types of genetic control (Fig. 6A, control, RNAi/+, GH146/+, NP225/+).

For the MB-specific RNAi expression, we used c747-Gal4 and OK107-Gal4 that drive expression in all MB lobes (Lee et al., 1999). MB-specific knockdown of dPQBP1 with the two drivers did not affect PI at $0 \mathrm{~h}$ (Fig. $6 \mathrm{~A}, \mathrm{MB}$ drivers). Again, the threshold of olfactory acuity was normal in the MB-specific APQBP1-RNAi flies (Fig. 6B). The efficiency of the knockdown by dPQBP1RNAi construct was validated by quantitative real-time PCR (supplemental Fig. 2, available at www.jneurosci.org as supplemental material). These results indicated that $\mathrm{CPQBP} 1$ does not affect learning acquisition via MB.

\section{NR1 mediates learning disturbance of PQBP1-mutant flies}

Next, we asked for the molecular mechanism of the learning disturbance in $\mathrm{APQBP} 1$-mutant flies. We previously found that the WWD of PQBP1 interacts with the C-terminal domain of the large subunit of RNA polymerase II and that PQBP1 affects gene expression at the transcription level (Okazawa et al., 2002). We also found that PQBP1 interacts with a splicing factor U5-15KD (Waragai et al., 2000; Zhang et al., 2000); thus, PQBP1 may affect gene expression through posttranscriptional regulation. Therefore, we suspected that repression of a certain target gene in PNs of the dPQBP1-mutant flies might lead to learning disturbance. NMDARs were considered to be a good candidate for the target gene of $\mathrm{dPQBP} 1$, since expression of most memory genes is restricted to MBs, whereas NMDARs are widely expressed (for review, see Keene and Waddell, 2007), and an ion channel-type receptor would account for such a short-term plasticity. Therefore, we were prompted to test whether $\mathrm{dNR} 1$ might be responsible for $0 \mathrm{~h}$ memory impairment in our dPQBP1-mutant flies.

First, we tested whether NMDAR protein expression is changed in dPQBP1-mutant flies by immunohistochemistry (Fig. 7A) and found dNR1 subunit of NMDAR but not dNR2 was decreased in dPQBP1-mutant flies (Fig. 7A; supplemental Fig. 3, available at www.jneurosci.org as supplemental material). Quantitative analysis of the signal intensities in ALs supported the reduction of $\mathrm{dNR} 1$ (Fig. $7 B$ ). Western blot analysis with wholehead tissues also confirmed the decrease of dNR1 protein in dPQBP1-mutant flies (Fig. 7C).

To test whether the reduction of dNR1 directly links to learning disturbance of dPQBP1-mutant flies, we reexpressed dNR1 in homozygous dPQBP1-mutant flies by using UAS-dNR1 flies. In the UAS-dNR1; homozygous dPQBP1-mutant flies, dNR1 was driven by GAL4 from piggyBac under the control of dPQBP1 endogenous enhancer/promoter. We found that expression level of dNR1 was recovered in PNs of the rescue flies by immunohistochemistry (data not shown), dNR1 was overexpressed in fly heads by Western blot analysis (Fig. $8 B$ ), and the dNR1 overexpression rescued the learning disturbance (Fig. $8 A$ ). In contrast to the previous observation that overexpression of dNR 1 in ellipsoid body enhanced LTM (Wu et al., 2007), PN-specific overexpression of dNR1 by GH146-Gal4 or NP225 drivers did not increase learning acquisition in normal flies (supplemental Fig. $4 A, B$, available at www.jneurosci.org as supplemental material). These results collectively support that $\mathrm{dNR} 1$ reduction in PNs is the direct cause of the learning disturbance of dPQBP1-mutant flies.

\section{Pharmacological rescue of dPQBP1-mutant phenotypes}

We then examined pharmacological rescue of the learning disturbance in $\mathrm{dPQBP} 1$-mutant flies. $\mathrm{LiCl}$ and a noncompetitive antagonist of metabotropic glutamate receptor (mGluR), 2-methyl6-(phenylethynyl) pyridine (MPEP), were reported to restore courtship memory and $\mathrm{MB}$ defects in a fly model of fragile $\mathrm{X}$ syndrome (McBride et al., 2005). Thus, we tested the effects of $\mathrm{LiCl}$ and MPEP on the learning defect of dPQBP1 mutants. However, neither $\mathrm{LiCl}$ nor MPEP could restore the $0 \mathrm{~h}$ memory of 
dPQBP1 mutants (Fig. 8C,D), indicating that the learning phenotype of our fly model is distinct from that in the fly model of fragile $\mathrm{X}$ syndrome. Although the fly learning system is not directly applicable to human learning, the result might suggest that the mGluR-mediated mechanism is not shared between fragile $\mathrm{X}$ syndrome and PQBP1-linked MR.

Histone deacetylase (HDAC) inhibitors were considered to be the next candidate for the rescue of $\mathrm{dPQBP} 1$ mutants. HDAC inhibitors are known to activate transcription and restore the normal phenotype in some model animals of polyglutamine diseases, in which transcriptional disturbance has been implicated (McCampbell et al., 2001; Steffan et al., 2001; Hockly et al., 2003; Minamiyama et al., 2004). From this reason, we tested the effects of HDAC inhibitors on the learning defect of the PQBP1-mutant flies. Suberoylanilide hydroxamic acid (SAHA) and phenylbutyrate counteracted by $\mathrm{NaOH}$ (PBA) partially restored the $0 \mathrm{~h}$ memory of homozygous dPQBP1 mutants (Fig. 8E,F). The restored learning was observed at an intermediate concentration, whereas the rescue effect was declined at a high concentration (Fig. $8 E, F$ ) presumably because both $\mathrm{PBA}$ and SAHA were toxic for flies at higher concentrations (data not shown). The similar reduction of the rescue effect at a high concentration was reported in the therapeutic use of HDAC inhibitor for a polyglutamine disease mouse model (Minamiyama et al., 2004). It is also of note that aversive olfactory conditioning is very sensitive to circumstantial factors even under the strict control of temperature and humidity; we had to repeat control experiments in each case; thus, the PIs of control and dPQBP1mutant flies (Fig. 8) were different from these in Figure $1 D$.

\section{Discussion}

In this study, we intended to analyze pathological basis of MR linked to PQBP1 mutations from the aspect of Drosophila model and found that hypomorph $\mathrm{APQBP} 1$ mutation impairs learning acquisition. PN-specific RNAi experiments support that the impaired learning acquisition is based on the reduced function of PQBP1 in PNs. Morphological analysis and temporal rescue experiment support the idea that $\mathrm{CPQBP} 1$ maintains postdevelopmental neuronal function critical for learning acquisition. The reduced expression of $\mathrm{dNR} 1$ in ALs and the recovery of learning defect by overexpressed dNR1 suggest that dPQBP1 controls learning acquisition in aversive olfactory conditioning via dNR1 expression at PNs.

\section{PQBP1 regulates $\mathrm{PN}$-dependent learning acquisition in} aversive olfactory conditioning

Olfactory memory circuits in Drosophila have been studied extensively, whereas the role of PNs in learning and memory remains unclear. Olfactory stimuli sensed at antennae or maxillary palps excite olfactory receptor neurons that connect to ALs. PNs transfer olfactory information from ALs to MBs and lateral horn.
MBs are generally believed to be a center of olfactory memory where many memory genes are expressed (for review, see Keene and Waddell, 2007), and PN has not been focused on before the work by the Davies group showing that dynamic modification of synaptic transmission of PNs may occur in classical aversive olfactory conditioning (Yu et al., 2004).

Thereafter, expression of rutabaga in PNs was shown to be essential for appetitive memory formation, but not for aversive memory formation (Thum et al., 2007; Blum et al., 2009). In addition, it was reported that expression level of the SDE3 helicase Armitage (Armi), a component of RISC (RNA-induced silencing complex) pathway is changed in PNs (GH146-positive neurons) in a glomerulus-specific manner after spaced training (Ashraf et al., 2006). Because armi mutant shows impaired LTM, a function of PNs in LTM seems to be abnormal. The armi mutant phenotype was successfully rescued by pan-neural expression of armi driven by elav-Gal4, whereas PN-specific rescue experiments have not been performed. The armi mutant did not show a defect in STM (Ashraf et al., 2006). In this regard, it is interesting that the $\mathrm{CPQBP} 1-$ mutant flies seem to show a larger extent of decline in $24 \mathrm{~h}$ memory (wild type, $48 \pm 2.3$, vs dPQBP1 mutant, $28 \pm 2.4$; mean $\pm \mathrm{SE} ; n=8$ ) and that human PQBP1 overexpression driven by a pan-neural driver, elav, induced LTM defect (Yoshimura et al., 2006). We tested whether MB-specific knockdown of dPQBP1 by RNAi affects $1 \mathrm{~h}$ memory, to evaluate the possible effect of dPQBP1 in MB neurons on 24 h memory after five spaced trainings. However, PI values at $1 \mathrm{~h}$ were not different between driver-only controls and RNAi flies (supplemental Fig. $4 C$, available at www.jneurosci.org as supplemental 


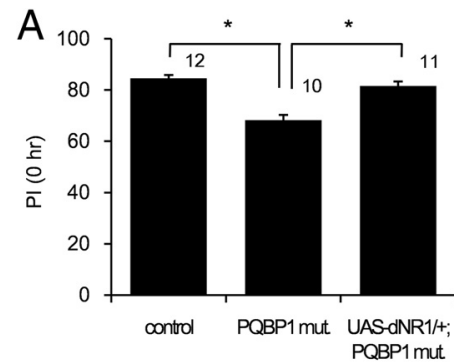

C

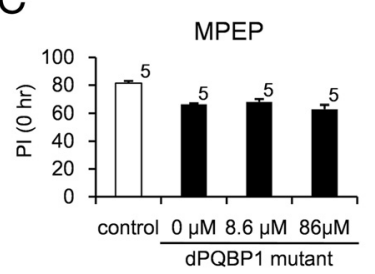

E

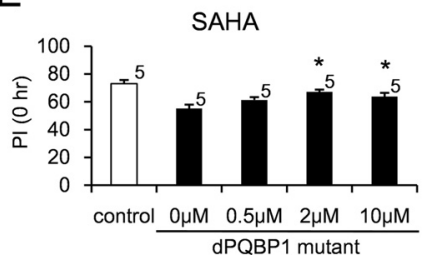

B

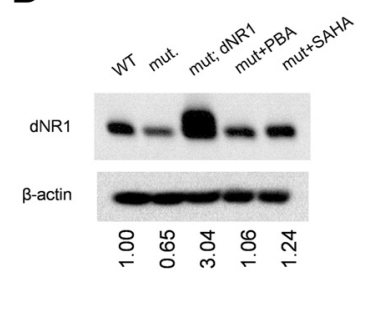

D

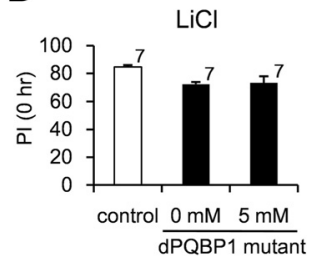

F

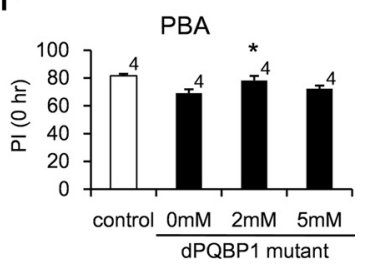

Figure 8. Genetic and pharmacological rescues of $\mathrm{PQBP} 1$-mutant flies. $A$, Overexpression of NR1 rescues learning defect of homozygous PQBP1-mutant flies at $0 \mathrm{~h}$. The asterisks indicate significant differences ( $p<0.05$, Welch's $t$ test). The numbers of experiments are shown above each bar. $\boldsymbol{B}$, Western blot analysis showed reduction and rescue of dNR1 expression in $\mathrm{DPQBP} 1$ mutant and UAS-dNR1; PQBP1-mutant flies, respectively. HDAC inhibitors slightly recovered dNR1 expression in correspondence with their mild effects on the learning impairment of dPQBP1-mutant flies (mut + SAHA and mut +PBA). The dNR1 signal intensities corrected by $\beta$-actin are compared with WT, and the values are shown below the panel. $C-F$, HDAC inhibitors partially rescued learning impairment of homozygous $\mathrm{DPQBP} 1$ mutants. A metabotropic glutamate receptor antagonist, MPEP, and lithium chloride did not recover learning impairment $(\boldsymbol{C}, \boldsymbol{D})$. LiCl was toxic at concentrations $>5 \mathrm{~mm}$ (data not shown); thus, only the result at a concentration of $5 \mathrm{~mm}$ was shown. HDAC inhibitors, SAHA and PBA, partially rescued learning impairment $(\boldsymbol{E}, \boldsymbol{F})$. PBA was toxic at concentrations $>5 \mathrm{~mm}$ (data not shown). Pl at $0 \mathrm{~h}$ was measured in all the treatments in parallel. The drug concentrations in the meal are put below each graph. The asterisks indicate significant differences from nontreated control flies ( $p<$ 0.05 , Welch's $t$ test). The number of experiments is shown above each bar.

material), indicating that possible physiological change of $\mathrm{MB}$ was not the basis for the defect of $24 \mathrm{~h}$ memory in $\mathrm{dPQBP} 1$ mutant.

The dPQBP1 mutant had a defect in learning acquisition (Fig. $1 D)$. In contrast, the dPQBP1 mutant showed normal odor responses even at a very low concentration (Fig. $1 E$ ). The odor avoidance responses used in this test are an innate behavior. de Belle and Heisenberg (1994) have shown that chemical ablation of $\mathrm{MB}$ did not affect this experience-independent response but the flies were impaired in aversive olfactory conditioning, suggesting lateral horn is sufficient to trigger an innate avoidance behavior. However, elimination of GH146-positive neurons with tetanus toxin affected olfactory acuity of ethyl acetate, trans-2hexanol, 2-hexanol, and 1-octen-3-ol at a low concentration (Heimbeck et al., 2001), whereas blocking transmission with shibire in the GH146-positive neuron did not affect olfactory acuity (Schwaerzel et al., 2002) (benzaldehyde and OCT). Considering these facts, acuity tests using an innate behavior may not reflect the transmission of odor information from antenna to MBs exactly. Therefore, although the odor acuity was normal in

the test with dPQBP1 mutant, it is still possible that odor transmission by $\mathrm{PNs}$ underlies impairment of learning acquisition.

Moreover, expression of dPQBP1 during development was dispensable for learning acquisition (Fig. 4). The knockdown of dPQBP1 in PNs induced a similar learning defect (Fig. 6A). The NP225 and GH146 drivers, whose expression patterns are partially overlapping, do not cover entire population of adult PNs. However, when both drivers are combined, the reduction of learning performance from the control (GH146/NP225, $89.0 \pm$ 1.7; GH146;RNAi/NP225, $71.7 \pm 1.6$; mean $\pm \mathrm{SE} ; n=7)$ was not increased compared with single RNAi. The reduction of PI in RNAi flies driven by each driver (Fig. 6A) was already equivalent to that observed in PQBP1-mutant flies (Fig. 1D). Therefore, it might suggest that the learning defect is attributable to PNs expressing both NP225 and GH146. The residual learning might be attributable to noncovered PNs by either of Gal4 drivers or attributable to the function of certain gene(s) other than $\mathrm{APQBP} 1$ in PNs.

For the MB-specific RNAi expression, we used c747-Gal4 because disruption of neurotransmission by expressing shibire in c747-positive cells almost completely blocks olfactory memory (Dubnau et al., 2001), although c747 is expressed in $\alpha, \beta$, and $\gamma$ lobes but not in $\alpha^{\prime}$ and $\beta^{\prime}$ lobes. Therefore, to rule out the possibility that $\mathrm{dPQBP} 1$ dysfunction in $\alpha^{\prime}$ and $\beta^{\prime}$ lobes underlies the learning defect, we also used OK107-Gal4 expressed in all MB lobes (Lee et al., 1999). In both drivers, MB-specific knockdown of APQBP1 did not induce a learning defect. These findings support the idea that postdevelopmental function of PNs regulates learning acquisition in olfactory aversive conditioning, in addition to the well known function of MBs (Yu et al., 2004). Although the importance of MB-APL neurons in learning was suggested (Liu et al., 2009), we have excluded the possible contribution of MB-APL neurons for APQBP1-mediated learning by using NP225 driver (Fig. 6) that does not induce expression in MB-APL neurons (Okada et al., 2009). Collectively, dPQBP1 is a novel gene regulating the $\mathrm{PN}$-dependent learning acquisition.

\section{Possible roles of PNs in learning acquisition}

A growing number of studies have postulated that PNs carry out an important computation that transforms the representation in AL to a sparser one in MB. This transformation is thought to underlie sensory coding in MBs (for review, see Masse et al., 2009). This is based on the premise Kenyon cells work as a coincident detector that is more selective with a higher spiking threshold (Perez-Orive et al., 2002; Murthy et al., 2008; Turner et al., 2008). Such highly selective Kenyon cells are considered to associate odor information and electric shock. Most memory genes are highly expressed in MBs (for review, see Keene and Waddell, 2007), supporting the idea that the association occurs at Kenyon cells.

However, synaptic plasticity of PNs after associative training was reported previously (Yu et al., 2004). The result suggests that PNs also associate odor and electric shock information. The simplest idea is that the association occurs at PNs independently from Kenyon cells. However, anatomical background of the association is not clear. Another idea is that plasticity of PNs enhances reliability of odor responses and thereby promotes the association between odor and electric shock information in Kenyon cells. In either case, aversive memory trace in PNs could be considered as learning acquisition. If the plasticity of PNs is reduced in APQBP1 mutant, the decline of learning acquisition can be explained well.

Other roles of PNs in learning acquisition might also be possible. They include "increasing the signal-to-noise ratio" (Bhandawat et al., 2007; Kazama and Wilson, 2008), "gain con- 
trol" (Bhandawat et al., 2007; Kazama and Wilson, 2008; Root et al., 2008), and "population coding" (Olsen et al., 2007). We could not completely exclude those possibilities in this study, and additional confirmation will be necessary.

\section{Molecular mechanisms of learning acquisition in PNs}

Morphological analyses of dPQBP1-downregulated fly showed reduced expression of $\mathrm{mCD} 8-\mathrm{GFP}$ and elav genes in PNs (Fig. $3 A, B)$ (our unpublished observation) without any obvious reduction of PNs in number (Fig. $3 C$ ). Given that mammalian PQBP1 is involved in both transcriptional regulation and the splicing machinery (Waragai et al., 2000; Okazawa et al., 2002), reduced gene expression at transcriptional and/or posttranscriptional levels in dPQBP1-mutant flies might be a cause for the impairment of learning acquisition. To support this idea, we found that SAHA and PBA, general upregulators of transcription, ameliorate the impairment of learning acquisition (Fig. 8).

For the aversive learning, short-term memory is believed to be stored in MBs because restricted expression of rutabaga to MBs fully rescued the loss of short-term memory in rutabaga mutant (Zars et al., 2000) and because expression of rutabaga in PNs by a $\mathrm{PN}$-specific driver, GH146-Gal4, fully rescues appetitive but not aversive short-term memory in rutabaga mutant (Thum et al., 2007; Blum et al., 2009). Meanwhile, the transmission of PNs was modified by an aversive electric shock (Yu et al., 2004). These findings collectively suggest that an unknown molecular pathway for aversive short-term memory might be located in PNs, and it is consistent with our hypothesis that dPQBP1 is involved in PNmediated learning acquisition.

Expression of dNR1 was reduced especially in ALs and surrounding areas of dPQBP1 mutant (Fig. $7 A, B$ ). In addition, overexpression of dNR1 rescued the learning defect in $\mathrm{dPQBP} 1$ mutant (Fig. $8 A$ ). Therefore, reduction of $\mathrm{dNR} 1$ seems to be an underlying mechanism of learning defect in dPQBP1-mutant flies. Interestingly, dNR1 expression outside of MBs was implicated in learning acquisition (Xia et al., 2005; Wu et al., 2007), which is consistent with our findings with $\mathrm{APQBP} 1$-mutant flies.

\section{Conclusion}

In conclusion, our study has shown that dysfunction of PNs in dPQBP1 mutants leads to an aversive learning defect. The dysfunction seems to reflect a decrease in $\mathrm{dNR} 1$. We propose that the $\mathrm{dPQBP} 1$ is a new gene involved in PN-dependent learning acquisition, and dPQBP1-mutant flies could be used as a tool for investigation of the $\mathrm{PN}$-dependent aversive memory.

\section{References}

Ashraf SI, McLoon AL, Sclarsic SM, Kunes S (2006) Synaptic protein synthesis associated with memory is regulated by the RISC pathway in Drosophila. Cell 124:191-205.

Berdnik D, Chihara T, Couto A, Luo L (2006) Wiring stability of the adult Drosophila olfactory circuit after lesion. J Neurosci 26:3367-3376.

Bhandawat V, Olsen SR, Gouwens NW, Schlief ML, Wilson RI (2007) Sensory processing in the Drosophila antennal lobe increases reliability and separability of ensemble odor representations. Nat Neurosci 10:1474-1482.

Blum AL, Li W, Cressy M, Dubnau J (2009) Short- and long-term memory in Drosophila require cAMP signaling in distinct neuron types. Curr Biol 19:1341-1350.

Busch A, Engemann S, Lurz R, Okazawa H, Lehrach H, Wanker EE (2003) Mutant huntingtin promotes the fibrillogenesis of wild-type huntingtin: a potential mechanism for loss of huntingtin function in Huntington's disease. J Biol Chem 278:41452-41461.

Chiang AS, Liu YC, Chiu SL, Hu SH, Huang CY, Hsieh CH (2001) Three- dimensional mapping of brain neuropils in the cockroach, Diploptera punctata. J Comp Neurol 440:1-11.

de Belle JS, Heisenberg M (1994) Associative odor learning in Drosophila abolished by chemical ablation of mushroom bodies. Science 263:692-695.

Dubnau J, Grady L, Kitamoto T, Tully T (2001) Disruption of neurotransmission in Drosophila mushroom body blocks retrieval but not acquisition of memory. Nature 411:476-480.

Dura JM, Preat T, Tully T (1993) Identification of linotte, a new gene affecting learning and memory in Drosophila melanogaster. J Neurogenet 9:1-14.

Heimbeck G, Bugnon V, Gendre N, Keller A, Stocker RF (2001) A central neural circuit for experience-independent olfactory and courtship behavior in Drosophila melanogaster. Proc Natl Acad Sci U S A 98:15336-15341.

Heisenberg M (2003) Mushroom body memoir: from maps to models. Nat Rev Neurosci 4:266-275.

Hockly E, Richon VM, Woodman B, Smith DL, Zhou X, Rosa E, Sathasivam K, Ghazi-Noori S, Mahal A, Lowden PA, Steffan JS, Marsh JL, Thompson LM, Lewis CM, Marks PA, Bates GP (2003) Suberoylanilide hydroxamic acid, a histone deacetylase inhibitor, ameliorates motor deficits in a mouse model of Huntington's disease. Proc Natl Acad Sci U S A 100:2041-2046.

Horn C, Offen N, Nystedt S, Häcker U, Wimmer EA (2003) piggyBac-based insertional mutagenesis and enhancer detection as a tool for functional insect genomics. Genetics 163:647-661.

Jefferis GS, Marin EC, Stocker RF, Luo L (2001) Target neuron prespecification in the olfactory map of Drosophila. Nature 414:204-208.

Kalscheuer VM, Freude K, Musante L, Jensen LR, Yntema HG, Gécz J, Sefiani A, Hoffmann K, Moser B, Haas S, Gurok U, Haesler S, Aranda B, Nshedjan A, Tzschach A, Hartmann N, Roloff TC, Shoichet S, Hagens O, Tao J, et al. (2003) Mutations in the polyglutamine binding protein 1 gene cause X-linked mental retardation. Nat Genet 35:313-315.

Kazama H, Wilson RI (2008) Homeostatic matching and nonlinear amplification at identified central synapses. Neuron 58:401-413.

Keene AC, Waddell S (2007) Drosophila olfactory memory: single genes to complex neural circuits. Nat Rev Neurosci 8:341-354.

Kleefstra T, Franken CE, Arens YH, Ramakers GJ, Yntema HG, Sistermans EA, Hulsmans CF, Nillesen WN, van Bokhoven H, de Vries BB, Hamel BC (2004) Genotype-phenotype studies in three families with mutations in the polyglutamine-binding protein 1 gene (PQBP1). Clin Genet 66:318-326.

Lai SL, Awasaki T, Ito K, Lee T (2008) Clonal analysis of Drosophila antennal lobe neurons: diverse neuronal architectures in the lateral neuroblast lineage. Development 135:2883-2893.

Lee T, Lee A, Luo L (1999) Development of the Drosophila mushroom bodies: sequential generation of three distinct types of neurons from a neuroblast. Development 126:4065-4076.

Lenski C, Abidi F, Meindl A, Gibson A, Platzer M, Frank Kooy R, Lubs HA, Stevenson RE, Ramser J, Schwartz CE (2004) Novel truncating mutations in the polyglutamine tract binding protein 1 gene (PQBP1) cause Renpenning syndrome and X-linked mental retardation in another family with microcephaly. Am J Hum Genet 74:777-780.

Lin HH, Lai JS, Chin AL, Chen YC, Chiang AS (2007) A map of olfactory representation in the Drosophila mushroom body. Cell 128:1205-1217.

Lin WY (2005) NMDA receptors are required in memory formation in Drosophila mushroom body. Biochem Biophys Res Commun 334:779-786.

Liu X, Buchanan ME, Han KA, Davis RL (2009) The GABA receptor RDL suppresses the conditioned stimulus pathway for olfactory learning. J Neurosci 29:1573-1579.

Margulies C, Tully T, Dubnau J (2005) Deconstructing memory in Drosophila. Curr Biol 15:R700-R713.

Masse NY, Turner GC, Jefferis GS (2009) Olfactory information processing in Drosophila. Curr Biol 19:R700-R713.

McBride SM, Choi CH, Wang Y, Liebelt D, Braunstein E, Ferreiro D, Sehgal A, Siwicki KK, Dockendorff TC, Nguyen HT, McDonald TV, Jongens TA (2005) Pharmacological rescue of synaptic plasticity, courtship behavior, and mushroom body defects in a Drosophila model of fragile X syndrome. Neuron 45:753-764.

McCampbell A, Taye AA, Whitty L, Penney E, Steffan JS, Fischbeck KH (2001) Histone deacetylase inhibitors reduce polyglutamine toxicity. Proc Natl Acad Sci U S A 98:15179-15184.

McGuire SE, Le PT, Osborn AJ, Matsumoto K, Davis RL (2003) Spatiotem- 
poral rescue of memory dysfunction in Drosophila. Science 302: $1765-1768$.

Minamiyama M, Katsuno M, Adachi H, Waza M, Sang C, Kobayashi Y, Tanaka F, Doyu M, Inukai A, Sobue G (2004) Sodium butyrate ameliorates phenotypic expression in a transgenic mouse model of spinal and bulbar muscular atrophy. Hum Mol Genet 13:1183-1192.

Murthy M, Fiete I, Laurent G (2008) Testing odor response stereotypy in the Drosophila mushroom body. Neuron 59:1009-1023.

Okada R, Awasaki T, Ito K (2009) Gamma-aminobutyric acid (GABA)mediated neural connections in the Drosophila antennal lobe. J Comp Neurol 514:74-91.

Okazawa H, Rich T, Chang A, Lin X, Waragai M, Kajikawa M, Enokido Y, Komuro A, Kato S, Shibata M, Hatanaka H, Mouradian MM, Sudol M, Kanazawa I (2002) Interaction between mutant ataxin-1 and PQBP-1 affects transcription and cell death. Neuron 34:701-713.

Olsen SR, Bhandawat V, Wilson RI (2007) Excitatory interactions between olfactory processing channels in the Drosophila antennal lobe. Neuron 54:89-103.

Perez-Orive J, Mazor O, Turner GC, Cassenaer S, Wilson RI, Laurent G (2002) Oscillations and sparsening of odor representations in the mushroom body. Science 297:359-365.

Pitman JL, DasGupta S, Krashes MJ, Leung B, Perrat PN, Waddell S (2009) There are many ways to train a fly. Fly (Austin) 3:3-9.

Poirier K, Lacombe D, Gilbert-Dussardier B, Raynaud M, Desportes V, de Brouwer AP, Moraine C, Fryns JP, Ropers HH, Beldjord C, Chelly J, Bienvenu T (2006) Screening of ARX in mental retardation families: consequences for the strategy of molecular diagnosis. Neurogenetics 7:39-46.

Restifo LL (2005) Mental retardation genes in Drosophila: new approaches to understanding and treating developmental brain disorders. Ment Retard Dev Disabil Res Rev 11:286-294.

Root CM, Masuyama K, Green DS, Enell LE, Nässel DR, Lee CH, Wang JW (2008) A presynaptic gain control mechanism fine-tunes olfactory behavior. Neuron 59:311-321.

Schwaerzel M, Heisenberg M, Zars T (2002) Extinction antagonizes olfactory memory at the subcellular level. Neuron 35:951-960.

Soller M, White K (2004) Elav. Curr Biol 14:R53.

Steffan JS, Bodai L, Pallos J, Poelman M, McCampbell A, Apostol BL, Kazantsev A, Schmidt E, Zhu YZ, Greenwald M, Kurokawa R, Housman DE, Jackson GR, Marsh JL, Thompson LM (2001) Histone deacetylase inhibitors arrest polyglutamine-dependent neurodegeneration in Drosophila. Nature 413:739-743.

Stevenson RE, Bennett CW, Abidi F, Kleefstra T, Porteous M, Simensen RJ, Lubs HA, Hamel BC, Schwartz CE (2005) Renpenning syndrome comes into focus. Am J Med Genet A 134:415-421.
Stocker RF, Heimbeck G, Gendre N, de Belle JS (1997) Neuroblast ablation in Drosophila P[GAL4] lines reveals origins of olfactory interneurons. J Neurobiol 32:443-456.

Tamura T, Chiang AS, Ito N, Liu HP, Horiuchi J, Tully T, Saitoe M (2003) Aging specifically impairs amnesiac-dependent memory in Drosophila. Neuron 40:1003-1011.

Thum AS, Jenett A, Ito K, Heisenberg M, Tanimoto H (2007) Multiple memory traces for olfactory reward learning in Drosophila. J Neurosci 27:11132-11138.

Tully T, Quinn WG (1985) Classical conditioning and retention in normal and mutant Drosophila melanogaster. J Comp Physiol A 157:263-277.

Tully T, Preat T, Boynton SC, Del Vecchio M (1994) Genetic dissection of consolidated memory in Drosophila. Cell 79:35-47.

Turner GC, Bazhenov M, Laurent G (2008) Olfactory representations by Drosophila mushroom body neurons. J Neurophysiol 99:734-746.

Waragai M, Lammers CH, Takeuchi S, Imafuku I, Udagawa Y, Kanazawa I, Kawabata M, Mouradian MM, Okazawa H (1999) PQBP-1, a novel polyglutamine tract-binding protein, inhibits transcription activation by Brn-2 and affects cell survival. Hum Mol Genet 8:977-987.

Waragai M, Junn E, Kajikawa M, Takeuchi S, Kanazawa I, Shibata M, Mouradian MM, Okazawa H (2000) PQBP-1/Npw38, a nuclear protein binding to the polyglutamine tract, interacts with U5-15kD/ $\operatorname{dim} 1 \mathrm{p}$ via the carboxyl-terminal domain. Biochem Biophys Res Commun 273:592-595.

Wu CL, Xia S, Fu TF, Wang H, Chen YH, Leong D, Chiang AS, Tully T (2007) Specific requirement of NMDA receptors for long-term memory consolidation in Drosophila ellipsoid body. Nat Neurosci 10:1578-1586

Xia S, Miyashita T, Fu TF, Lin WY, Wu CL, Pyzocha L, Lin IR, Saitoe M, Tully T, Chiang AS (2005) NMDA receptors mediate olfactory learning and memory in Drosophila. Curr Biol 15:603-615.

Yang MY, Armstrong JD, Vilinsky I, Strausfeld NJ, Kaiser K (1995) Subdivision of the Drosophila mushroom bodies by enhancer-trap expression patterns. Neuron 15:45-54.

Yoshimura N, Horiuchi D, Shibata M, Saitoe M, Qi ML, Okazawa H (2006) Expression of human PQBP-1 in Drosophila impairs long-term memory and induces abnormal courtship. FEBS Lett 580:2335-2340.

Yu D, Ponomarev A, Davis RL (2004) Altered representation of the spatial code for odors after olfactory classical conditioning; memory trace formation by synaptic recruitment. Neuron 42:437-449.

Zars T, Fischer M, Schulz R, Heisenberg M (2000) Localization of a shortterm memory in Drosophila. Science 288:672-675.

Zhang Y, Lindblom T, Chang A, Sudol M, Sluder AE, Golemis EA (2000) Evidence that dim 1 associates with proteins involved in pre-mRNA splicing, and delineation of residues essential for $\operatorname{dim} 1$ interactions with hnRNP F and Npw38/PQBP-1. Gene 257:33-43. 